\title{
Explaining historical change in terms of LHT: A pluralistic causal framework is needed
}

\author{
Aurélien Allarda and Antoine Marie ${ }^{b, c}$ \\ a Laboratory of Political Theory, University of Paris 8, 93526 Saint Denis, France; \\ ${ }^{\mathrm{b}}$ Center for Research and Interdisciplinarity; Paris Descartes University, 75004 Paris, \\ France; ' Institut Jean Nicod, ENS, EHESS, CNRS, PSL Research University, 75005 \\ Paris, France.
}

\begin{abstract}
Baumard suggests that the advent, through phenotypic plasticity mechanisms, of future-oriented preferences and creative mindsets in eighteenth century Great Britain explains the wave of innovations that drove the British Industrial Revolution. We argue that, although this approach is promising, Baumard's model would benefit from being supplemented by demographic, economic, and sociological explanations independent of Life History Theory (LHT).
\end{abstract}

Baumard proposes that the advent of future-oriented preferences and creative mindsets in eighteenth century Great Britain may be explained as genetically driven cognitive adjustments to environmental change. This set of preferences would then have caused the cascade of technological innovations characteristic of the Industrial Revolution.

We view Baumard's proposal as a bold and original contribution for at least two reasons. First, it convincingly suggests that a significant part of cultural stability within a 
given historical time period (i.e., eighteenth and nineteenth century England) may result from the influence of low-level "cognitive attractors" (viz., "slow" preferences and intuitions) on individuals' aspirations and conducts (Sperber 1996; Sperber \& Hirschfeld 2004). This attractionist causal framework is explicitly presented as an alternative to institutionalist approaches. But it also departs from models of cultural evolution focused on the influence of reflexive, culturally transmitted prescriptions (e.g., to cooperate, or to save one's money) that often suffer from the limitation of overestimating the motivational power of reflexive beliefs on individual conducts, without first asking the question of which underlying psychological substrate those norms require to enjoy cultural success (Norenzayan et al. 2016; Weber 2002).

Second, Baumard's framework is to be praised for exploring the idea that the jurisdiction of the evolutionary sciences is not limited to explaining fixed and stable traits, but also traits that vary between populations and over time. Changing ancestral ecologies and the adaptive trade-off between specialization and flexibility often selected for an array of potential behavioral reactions. Commitment to the existence of a universal biological predisposition is compatible with the recognition of it displaying important local variability. Conversely, cultural variability on a trait is not an argument against it having a potentially strong genetic basis. Emphasizing these points seems particularly important because of the frequent and unfortunate tendency to pit purely "biological" explanations against purely "cultural" ones, as if they were mutually exclusive.

Now, what remains unclear to us is the exact status Baumard attributes to phenotypic plasticity mechanisms within the wider causal account of the Industrial Revolution. Should the generalization of a "slower" mindset in eighteenth century England be considered a 
necessary cause of the revolution, a sufficient cause, or neither? Baumard's framework does not acknowledge any explanation aside from LHT, which suggests that he intends genetically driven change in preferences in response to environmental shift to be explanatorily sufficient. The issue, however, is that Holland was in the eighteenth century richer and more urbanized than England (Baumard sect. 4.2 and 4.3; Broadberry et al. 2015). If a "psychology of affluence" (sect. 3) developed in Great Britain, one would also expect it to have developed in Holland, and to have brought about similar consequences. Yet, it was in Great Britain that the Industrial Revolution took off. Therefore, phenotypic plasticity alone cannot account for the divergent development of these countries. We surmise that Baumard's narrative would benefit from being supplemented by more classical demographic, sociological, and economic considerations. We illustrate the importance of such factors by taking the example of population size, which we consider to be crucial.

Research based on both theoretical models and historical studies (Henrich 2004; Kremer 1993) has shown that technological progress increases as a linear function of population size. This stems from at least two reasons. First, ceteris paribus, the larger the population, the greater the likelihood of inventions being made. Second, technology and knowledge are nonrivalrous: A given innovation can be enjoyed by many consumers simultaneously without any additional cost. Thus, the average amount of goods and services enjoyed by each consumer increases in direct proportion to the total number of inventors and their output (Kremer 1993). Third, culture being cumulative, innovation is fueled by people's tendency to imitate and get inspiration from the most successful inventions available (Henrich 2004). Consequently, a larger population increases the probability of emergence of exceptionally creative individuals, whose innovations will be enjoyed, imitated, and improved by the rest of the population. 
Adding these demographic considerations to Baumard's model is all the more important because their force is magnified by a country's wealth and level of education. As Squicciarini and Voigtländer (2015) stressed, the upper class acquired a special importance for economic development at the onset of the Industrial Revolution because of its members' high level of knowledge and wealth. Independent of LHT mechanisms, these assets enabled the upper class to afford technical education, to dedicate free time to technological exploration, and to stay constantly informed of the latest innovations.

Great Britain, at the dawn of the Industrial Revolution, combined two factors: a high level of economic prosperity and a large population. As Baumard showed (sect. 4.2), other populous European countries, such as France and Germany, were much poorer. Holland, one of the only other countries that rivaled England's prosperity (Broadberry et al. 2015), had only 2 million people in contrast to Britain's 6 million (or 9 million, if one includes Ireland; Broadberry et al. 2015; Livi Bacci 2000). The conjunction of high prosperity and demography would have enabled the British economy to benefit from a pool of innovators both exceptionally large and affluent.

We do not want to suggest that the size of such alternative mechanisms would have been massive. What we want to argue is that they should be taken into account, as plausible and parsimonious complements to LHT. There is no doubt that traditional historical research has neglected evolved psychological dispositions, that cognitive attraction accounts are a major achievement of the naturalistic social sciences, and that LHT is an interesting tool for explaining diachronic and synchronic variation. But Baumard, in turn, seems to be minimizing the importance of demographic and sociological factors. Pendular oscillations 
between sharply contrasted explanatory models are recurrent throughout the history of science. However, theoretical accuracy culminates in interdisciplinary syntheses that manage to do full justice to the causal complexity of history. Baumard's theory provides the basis for an even more exact and inclusive model of the origins of the Industrial Revolution that would eschew the temptation of causal reductionism.

\section{References}

Broadberry, S., Campbell, B. M. S., Klein, A., Overton M. \& van Leeuwen, B. (2015) British Economic Growth 1270-1870. Cambridge University Press.

Henrich, J. (2004) Demography and cultural evolution: How adaptive cultural processes can produce maladaptive losses: The Tasmanian case. American Antiquity 69(2):197-214. Available at: https://doi.org/10.2307/4128416.

Kremer, M. (1993) Population growth and technological change: One million B.C. to 1990. The Quarterly Journal of Economics 108(3):681-716. Available at: https://doi.org/10.2307/2118405.

Livi Bacci, M. (2000) The population of Europe. Wiley-Blackwell.

Norenzayan, A., Shariff, A. F., Gervais, W. M., Willard, A. K., McNamara, R. A., Slingerland, E. \& Henrich, J. (2016) The cultural evolution of prosocial religions. Behavioral and Brain Sciences 39:e1.

Available at: https://doi.org/10.1017/S0140525X14001356.

Sperber, D. (1996) Explaining culture: A naturalistic approach, first edition, vol. 323. Blackwell.

Sperber, D. \& Hirschfeld, L. A. (2004) The cognitive foundations of cultural stability and diversity. Trends in Cognitive Sciences 8(1):40-46. 
Available at: https://doi.org/10.1016/j.tics.2003.11.002

Squicciarini, M. P. \& Voigtländer, N. (2015) Human capital and industrialization: Evidence from the Age of Enlightenment. The Quarterly Journal of Economics 130(4):1825-83. Available at: https://doi.org/10.1093/qje/qjv025

Weber, M. (2002) The Protestant ethic and the spirit of capitalism: And other writings. Penguin Classics. 